Ann. Zootech., I974, 23 (2), 23I-236.

NOTE

\title{
COMPOSITION EN ACIDES AMINÉS DES PROTÉINES CORPORELLES DU PORCELET ENTRE LA NAISSANCE ET L’ÂGE DE HUIT SEMAINES
}

\author{
A. AUMAITRE et P. H. DUÉE \\ avec la collaboration technique de J. Chevalier, J. Jung et J. Peiniau \\ Station de Recherches sur l'Élevage des Porcs, \\ Centre national de Recherches zootechniques, I. N. R. A., \\ 78350 Jouy en Josas
}

\section{RÉSUMÉ}

La composition en acides aminés des protéines du porcelet entre la naissance et l'âge de huit semaines a été déterminée, par dosage chromatographique sur un échantillon moyen effectué à 7 stades, à partir d'un effectif de 42 animaux de race Large White.

Les teneurs moyennes, exprimées en g pour $\mathrm{i} 6 \mathrm{~g}$ d'azote et ne tenant pas compte des valeurs obtenues à la naissance, sont les suivantes :

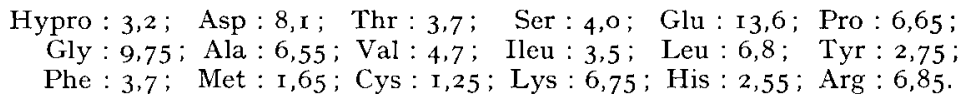

Par rapport aux valeurs moyennes présentées ci-dessus, l'examen de la composition en acides aminés des protéines du porcelet à la naissance fait apparaître une diminution de la somme des acides aminés indispensables (lysine, histidine, isoleucine, leucine, méthionine) d'une part, et une augmentation de certains acides aminés non indispensables (hydroxyproline, glycine, proline) d'autre part. L'évolution des dépôts corporels en matières azotées et en certains acides aminés indispensables est également présentée.

\section{INTRODUCTION}

La croissance postnatale du porcelet se révèle particulièrement rapide : l'animal multiplie son poids de naissance par quinze à vingt en l'espace de 2 mois. Les différents facteurs de variation du gain de poids au cours de cette période ont été analysés récemment (Aumaitre et al., 
1966). Il apparaît, d'une manière générale, que la vitesse de croissance passe par un minimum entre le $\mathrm{I}_{\mathrm{I}}{ }^{\mathrm{e}}$ et le $\mathbf{r} 7^{\mathbf{e}}$ jour. Les explications de cette "crise ", quoique multiples, ne sont pas satisfaisantes.

Les besoins nutritionnels, liés au développement important de l'animal, notamment les besoins azotés quantitatif et qualitatif durant cette phase de la croissance, sont encore mal connus (Aumaitre et Sève, 1973). L'application des méthodes de sevrage précoce implique cependant la connaissance précise des dépenses de croissance du porcelet. L'examen des modifications de la composition chimique corporelle peut, dans le cas présent, lever partiellement les imprécisions signalées précédemment. Les résultats rapportés concernent l'évolution des teneurs corporelles du porcelet en matières azotées et en acides aminés, depuis sa naissance jusqu'à l'âge de huit semaines.

\section{MATÉRIEL, ET MÉTHODES}

L'étude a été entreprise sur 42 porcelets allaités (19 mâles et 23 femelles), de race Large White. On réalise une répartition au hasard des animaux à la naissance et l'on prélève 6 porcelets (mâles et femelles) homogènes et représentatifs en poids aux stades considérés (naissance, 7, I4, 2I, 28, 42 et 56 jours environ).

L'âge moyen des animaux à chaque stade de l'étude et leur poids vif sont consignés dans le tableau $\mathrm{I}$, ainsi que l'évolution du dépôt corporel de protéines $(N \times 6,25)$, déterminé analytiquement.

\section{TABLEAU I}

Caractéristiques des porcelets à chaque stade d'abattage

Nombre d'animaux à chaque stade : 6

\begin{tabular}{|c|c|c|c|c|c|c|c|}
\hline Stade d'abattage & Naissance & $\begin{array}{c}1^{\text {re }} \\
\text { semaine }\end{array}$ & $\begin{array}{c}2^{\mathrm{e}} \\
\text { semaine }\end{array}$ & $\begin{array}{c}3^{\mathbf{e}} \\
\text { semaine }\end{array}$ & $\begin{array}{c}4^{\mathbf{e}} \\
\text { semaine }\end{array}$ & $\begin{array}{c}6^{\mathrm{e}} \\
\text { semaine }\end{array}$ & $\begin{array}{c}8^{e} \\
\text { semaine }\end{array}$ \\
\hline Poids vif moyen $(\mathrm{kg})$ & 1,28 & 2,32 & 3,47 & 5,47 & 6,36 & 10,50 & 16,25 \\
\hline Poids vif vide moy. $(\mathrm{kg}) .$. & 1,18 & 2,14 & 3,13 & 4,97 & 5,62 & 9,47 & 14,73 \\
\hline $\begin{array}{l}\text { Age moyen d'abattage } \\
\text { (jours) } \ldots \ldots \ldots \ldots \ldots\end{array}$ & 0 & 7,2 & 14,8 & 22,3 & 28,8 & 42,7 & 57,8 \\
\hline $\begin{array}{l}\text { Quantités de matières azo- } \\
\text { tées }(\mathrm{N} \times 6,25) \text { en } \mathrm{g} / \text { por- } \\
\text { celet vide } \ldots \ldots \ldots \ldots \ldots\end{array}$ & 150 & 287 & 510 & 760 & 967 & 1490 & 2458 \\
\hline
\end{tabular}

Les animaux sont sacrifiés le matin après pesée, sans jeûne préalable; le sang est recueilli quantitativement, le tube digestif est vidé de son contenu, ainsi que la vésicule biliaire et la vessie. Après séparation en 3 fractions, la carcasse, le sang et les viscères sont congelés à $-20^{\circ} \mathrm{C}$ pendant au moins 8 jours, puis broyés finement. Un échantillon représentatif pour chaque animal (200 g) et par fraction est lyophilisé pendant 24 heures. Après reconstitution de l'ensemble du porcelet par un mélange pondéré d'une quantité aliquote des fractions, on réalise une délipidation par extraction à chaud à l'alcool puis à l'éther éthyliques dans un appareil de Soxhlet. Un seul échantillon final par stade, de 50 grammes environ, est préparé en le broyant successivement en présence de neige carbonique, puis dans un broyeur à billes.

Les teneurs en acides aminés des porcelets sont déterminées sur l'échantillon lyophilisé et délipidé, après hydrolyse acide, suivant la méthode de MOORE et al. (1958), utilisant la technique de chromatographie sur colonne échangeuse d'ions. Le tryptophane, totalement détruit lors de l'hydrolyse acide, n'est pas dosé ; les acides aminés soufrés sont oxydés au préalable par l'acide performique et dosés par la même méthode que précédemment. 


\section{RÉSULTATS E'T DISCUSSION}

La composition en acides aminés des protéines du porcelet figure dans le tableau 2. Si l'on compare cette analyse à la composition des protéines musculaires du porc déterminé par PIon et FAUCONNEAU ( 1966 ), il ressort cependant une teneur plus faible de l'ensemble des acides aminés indispensables, la lysine, la thréonine, l'isoleucine et la méthionine en particulier dans le cas des protéines du porcelet. Par contre, certains acides aminés non indispensables, I'hydroxyproline, la proline et la glycine, sont en quantités plus importantes. Ceci reflète, dans l'échantillon analysé la présence des protéines d'os et de collagène, en plus de celles du tissu musculaire. Ces résultats sont, dans l'ensemble, en accord avec ceux de Williams et al. (i954) et de Shmanenkov (1967), à l'exception des teneurs en lysine et en valine, plus élevées dans la première analyse et des teneurs en acides aminés soufrés et aromatiques, plus élevées dans la seconde. Ils diffèrent toutefois, pour les teneurs à la naissance, de ceux de GRUHN (1965) obtenus dans d'autres conditions analytiques.

\section{TABLEAU 2}

Composition en acides aminés des protéines du porcelet suivant l'âge d'abattage (en $\mathrm{g} / \mathrm{r} 6 \mathrm{~g} \mathrm{~N}$ )

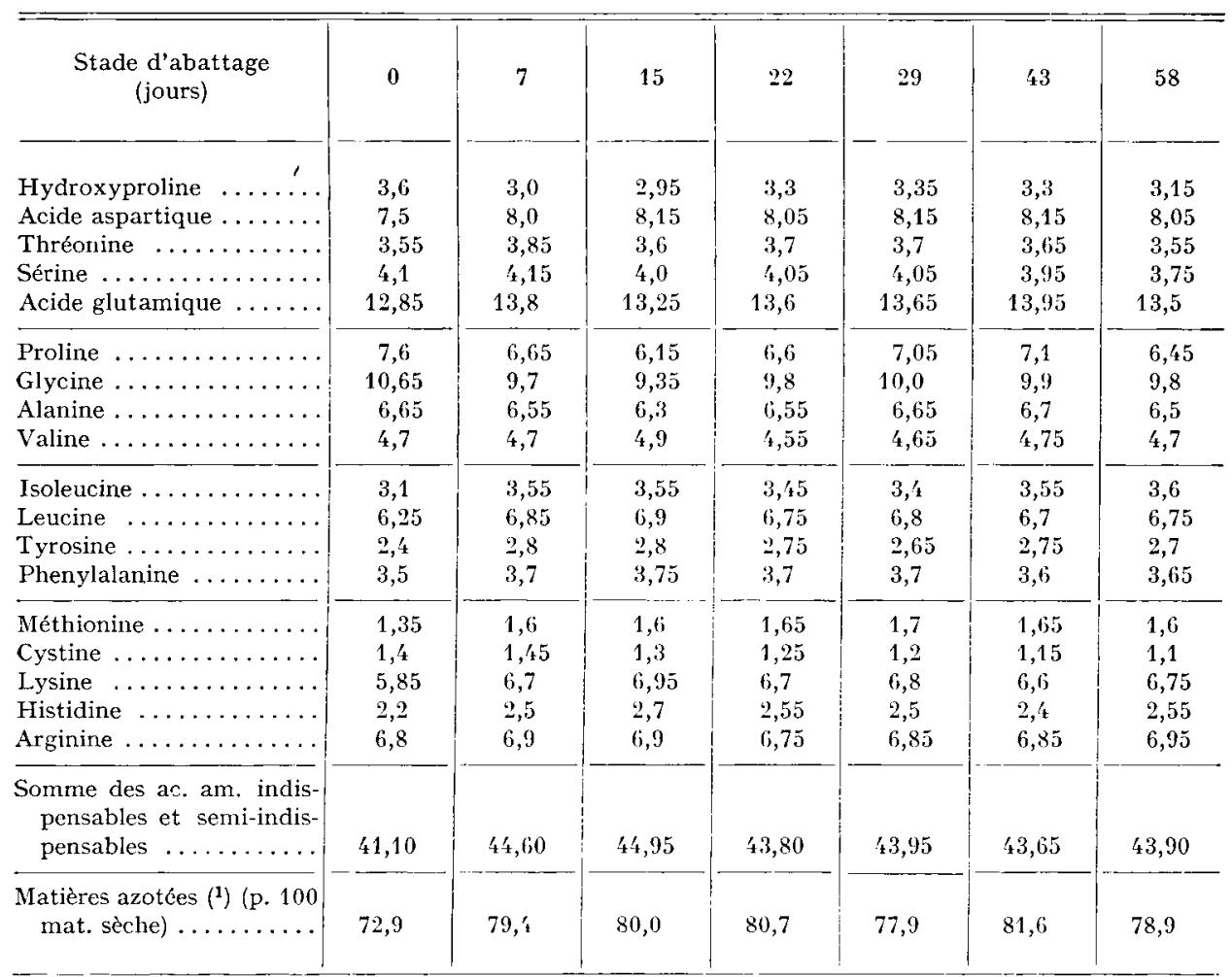

(1) Échantillon lyophilisé et dégraissé. 
La composition des protéines da porcelet montre une évolution différente suivant l'âge de l'animal et l'acide aminé considéré. La somme des acides aminés indispensables et semi-indispensables par rapport à la somme des acides aminés augmente entre la naissance et la seconde semaine; cette augmentation étant plus marquée pendant les premiers jours de la vie du porcelet; après la deuxième semaine, la composition des protéines ne semble plus modifiée par l'âge de l'animal, si l'on considère les résultats jusqu'à la huitième semaine. Cette évolution concerne surtout, parmi les acides aminés indispensables, la lysine, l'histidine, l'isoleucine et la leucine et à un moindre degré, la tyrosine. Par contre, les teneurs en arginine, thréonine, valine et phénylalanine des protéines du porcelet ne subissent pas de modification suivant les différents stades étudiés. Enfin, l'étude du rapport des teneurs en cystine et méthionine montre une analogie avec l'étude correspondante dans le lait de truie (DUÉE et JuNG, 1973). Si ce rapport cystine/méthionine est supérieur à I à la naissance, comme pour le colostrum, il diminue ensuite et reste inférieur à l'unité, comme pour le lait de truie. En ce qui concerne les acides aminés non indispensables, il est à noter surtout une diminution des teneurs en hydroxyproline, glycine et proline entre la naissance et la première semaine. D'autre part, les concentrations en acide aspartique et en acide glutamique augmentent légèrement entre ces deux stades. A partir de la seconde semaine, il ne semble plus y avoir de modification des teneurs en différents acides aminés non indispensables.

Il est vraisemblable que l'augmentation importante de certains acides aminés indispensables pendant les premiers jours de la vie du porcelet, parallèle à une diminution des acides aminés non indispensables abondants dans les protéines des tissus de soutien (hydroxyproline, glycine) traduise une évolution rapide des proportions des différents tissus du porcelet, en particulier une augmentation relative du poids de carcasse et des tissus musculaires. Cette dernière hypothèse est justifiée par une étude de STRuNz (1965), montrant une augmentation, dans la proportion de tissus musculaires, de $\mathbf{I} 2,4$ p. Ioo entre la naissance et la première semaine d'âge et, parallèlement, une diminution dans les proportions d'os de 27,9 p. Ioo et de peau de I6, I p. Ioo. D'après STRunz, cette évolution se poursuit, mais beaucoup plus lentement au cours des semaines suivantes. C'est ce qui apparaît dans la composition qualitative des protéines du porcelet (tabl. 2) qui ne montre pas de différences notables entre la $3^{\mathrm{e}}$ et la $8^{\mathrm{e}}$ semaine.

En termes de quantités déposées, les résultats illustrés par les graphiques de la figure I,

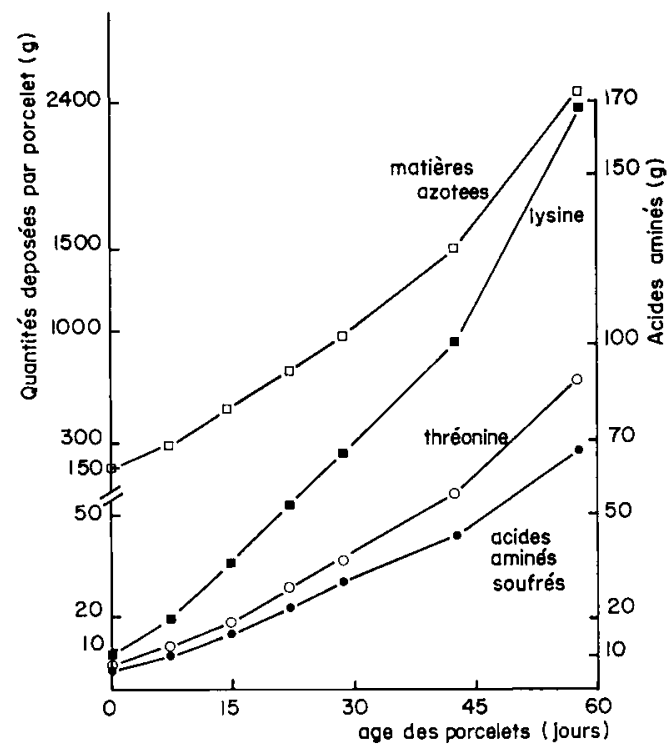

Fig. r. - Evolution des dépôts corporels en matières azotées et en certains acides aminés indispensables en fonction de l'âge 
qui tiennent compte des teneurs en matières azotées des porcelets aux différents stades d'abattages, mettent en évidence une augmentation des quantités corporelles d'acides aminés indispensables en fonction de l'âge de l'animal. Ainsi, on peut chiffrer le dépôt journalier en matières azotées et en acides aminés indispensables et semi-indispensables suivant 3 classes de poids vif des animaux (0-5 kg, 5-Io kg, IO-I $5 \mathrm{~kg}$ ). Considérant les deux classes extrêmes dans lesquelles la vitesse de croissance varie du simple au double, on constate que la teneur en protéines du gain de poids augmente avec l'âge. De plus, les protéines corporelles s'enrichissent surtout en acides aminés basiques (lysine, histidine, arginine) de même qu'en valine et isoleucine; par contre, elles s'appauvrissent en thréonine, tyrosine et acides aminés soufrés (tabl. 3).

\section{TABLEAU 3}

Évolution des dépôts d'acides aminés indispensables et semi-indispensables dans le porcelet au cours de la croissance (g par jour)

\begin{tabular}{|c|c|c|c|}
\hline \multirow{2}{*}{$\begin{array}{l}\text { Quantités } \\
\text { déposées en }\end{array}$} & \multicolumn{3}{|c|}{ Classe de poids $(\mathrm{kg})$} \\
\hline & $0-5$ & $5-10$ & $10-15$ \\
\hline$\ldots \ldots \ldots \ldots \ldots$ & 1,9 & 2,25 & 4,5 \\
\hline Histidine $\ldots \ldots \ldots \ldots \ldots$ & 0,75 & 0,8 & 1,8 \\
\hline Arginine $\ldots \ldots \ldots \ldots \ldots$ & 1,85 & 2,4 & 4,6 \\
\hline Thréonine ........... & 1,05 & 1,25 & 2,2 \\
\hline Valine ... & 1,25 & 1,7 & 3,0 \\
\hline Isoleucine . . . . . . . & 1,0 & 1,25 & 2,35 \\
\hline Leucine . . . . . . . . . . . . & 1,9 & 2,3 & 4,4 \\
\hline Tyrosine $\ldots \ldots \ldots \ldots \ldots$ & 0,8 & 0,95 & 1,7 \\
\hline Phénylalanine $\ldots \ldots \ldots \ldots$ & 1,05 & 1,2 & 2,4 \\
\hline Cystine $\ldots \ldots \ldots \ldots \ldots$ & 0,35 & 0,35 & 0,65 \\
\hline Méthionine $\ldots \ldots \ldots \ldots$ & 0,5 & 0,55 & 1,0 \\
\hline Matières azotées........ & 27,8 & 34,7 & 64,55 \\
\hline Gain de poids $\ldots \ldots \ldots \ldots$ & 190 & 240 & 383 \\
\hline
\end{tabular}

En conclusion, il apparaît que cette détermination de la composition des protéines corporelles peut servir de base comme le proposaient Williams et al. (I954) et Gruhn (I965) à une expérimentation directe sur le besoin en acides aminés du jeune porcelet à l'aide de méthodes complémentaires.

Reçu pour publication en février 1974.

\section{SUMMARY}

AMINO ACID COMPOSITION OF PIGIET BODY PROTEINS BETWEEN BIRTH AND 8 WEEKS OF AGE

The amino acid composition of the body proteins of suckled piglets from birth till the age of 8 weeks, was determined by chromatographic analysis of a mean sample taken at 7 different growth stages, from a herd of 42 Large White piglets, slaughtered at birth, 7, I 4, 21, 28, 42 and 56 days of age. 
The mean contents, expressed in $\mathrm{g} / \mathrm{I} 6 \mathrm{~g} \mathrm{~N}$ and without including new born values, were the following :

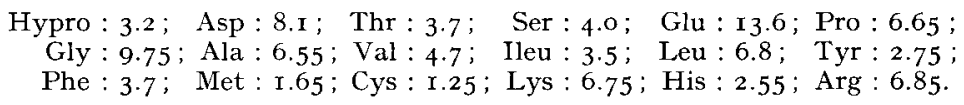

As related to the above indicated mean values, the examination of the amino acid composition of piglet proteins at birth showed, on the one hand, a decrease in the total amount of essential amino acids (lysine, histidine, isoleucine, leucine, méthionine) and, on the other hand, an increase of some non essential amino acids (hydroxyproline, glycine, proline). Variation in the body depositions of crude protein and of some essential amino acids is also presented in this paper.

\section{RÉFÉRENCES BIBLIOGRAPHIQUES}

Aumaitre A., Legault C., Salmon-Legagneur E., 1966. Aspects biométriques de la croissance pondérale du Porcelet. I. Influence du sexe, de l'année de naissance, du numéro et de la taille de la portée. Ann. Zootech., 15, 3I3-33r.

Aumatre A., Seve B., 1973. Nutrition azotée du Porcelet, sevrage précoce et fonctionnel. Journées d'Information sur l'Alimentation azotée des animaux, I. N. R. A. éd., Paris, p. 43-49.

Dú́e P. H., Jung J., 1973. Composition en acides aminés du lait de truie. Ann. Zootech., 22, $243-247$.

GruhN K, 1965. Einfluss des Alters auf Nähstoffgehalt und Fleischeiweisszusammensetzung bei schweinen. Die Nährung, 9, 325-333.

Moore S., Spackman D. H., Stein W. H., I958. Chromatography of Amino Acids on sulfonated polystyrene resins. Anal. Chem., 30, i $185^{-1}$ I 90.

Pion R., Fauconneau G., I966. Les acides aminés des protéines alimentaires. Méthodes de dosage et résultats obtenus. Cahiers A.E.C., n० 6, 157-175.

Shmanenkov N. A., I967. Cité par Buraczewski S., 1972. Views on the pig's requirements for amino acids and new sources of these nutrients suitable for use in the feeding of pigs. Symposium F. A. O., Genève.

Strunz K., r965. Die relative N-Verteilung im Körper und der Nukleinsaüren. Gehalt einiger Gewebe beim wachsenden Schwein. Habilitationsschrift. Göttingen, I-224.

Williams H. H., Curtin L. V., Abraham J., Loosli J. K., Maynard L. A., i954. Estimation of growth requirements for amino acids by assay of the carcass. J. Biol. Chem., 208, 277-286. 